\title{
Lymph node assessment by diffusion weighted imaging in cervical cancer
}

\author{
Luis Martí-Bonmatí
}

Received: 17 November 2010 /Revised: 9 December 2010 /Accepted: 15 December 2010 /Published online: 6 January 2011

(C) European Society of Radiology 2011

The use of node size alone to determine the presence of metastatic lymphadenopathy has been shown to be a poor discriminator in pelvic oncological imaging. Functional imaging is becoming increasingly important in the evaluation of cancer patients because of the limitations of morphologic imaging. In patients with uterine cervical cancer, it is quite important to evaluate the involvement of lymph nodes as lymph nodes metastases are an important prognostic factor, correlating with survival and modifying treatment option $[1,2]$. In some institutions, routine laparoscopic lymphadenectomy is performed to accurately stage lymphatic extension. Non-invasive preoperative lymph nodes staging seems quite important in patient's management as treatment is heavily influenced according to the presence or absence of lymph node involvement.

Recent imaging strategies, such as diffusion-weighted MR (DW-MR) imaging and positron emission tomography and computed tomography (PET/CT) have lead to some controversies regarding the capability of preoperative imaging to accurately stage malignant lymphadenopathy. DW-MR imaging is a functional imaging technique whose contrast derives from the random motion of water molecules within tissues. PET/CT imaging contrast derives from the metabolic consumption of glucose within tissues.

DW-MR sequences are quite relevant and recent imaging techniques that are used to define disease involvement. In oncological imaging, DW-MR imaging has been linked to lesion aggressiveness and tumour response, although the biophysical basis for this is not completely understood. In

\section{Martí-Bonmatí $(\bowtie)$}

Department of Radiology,

La Fe University and Polytechnic Hospital,

Valencia, Spain

e-mail: Luis.Marti@uv.es our clinical experience with female pelvic cancers, both benign and metastatic lymph nodes may appear with high signal intensity on high $b$-value images, with corresponding low apparent diffusion coefficient (ADC) values. The proof of concept behind the paper by Choi at el [1] is that tumour involvement changes the diffusion properties of malignant lymph nodes as measured by ADC. The reasons for diffusion restriction and, therefore, lower ADC values in malignant nodes is probably related to a combination of higher cellularity, tissue disorganization and increased extracellular space tortuosity. If correct, lymph node involvement, both early in diagnosis and in the follow-up, could be evaluated with diffusion-weighted images and the corresponding ADC calculations. If these changes can be used to predict staging and monitor early cancer treatment response, DW-MR techniques will become a considerable benefit for these patients.

Choi et al. evaluated, in a node-by-node comparison, the diffusion-weighted ADC calculation and size-based criteria on $\mathrm{T} 2$-weighted images with PET/CT findings in a large series of patients [1]. The series included 163 patients with 339 pelvic lymph nodes with short-axis diameter $>5 \mathrm{~mm}$. The minimum and mean ADC, shortand long-axis diameters, and ratio of long- to short-axis diameters (L/S ratio) were compared in PET/CT-positive and -negative lymph nodes. There were 118 (35\%) positive nodes in 58 patients. There were statistically different values in PET/CT-positive and PET/CT-negative groups in minimum and mean ADCs, short- and long-axis diameters, and $\mathrm{L} / \mathrm{S}$ ratio $(P<0.05)$. The best discriminator was the minimum ADC, with a larger area-under-thecurve $(\mathrm{Az}=0.864)$ than mean $\mathrm{ADC}(0.836)$, short-axis diameter $(0.764)$, long-axis diameter $(0.640)$ and $\mathrm{L} / \mathrm{S}$ ratio (0.652). The sensitivity and accuracy of the minimum ADC $(86 \%, 82 \%)$ were also greater than when the mean ADC value was used. 
The paper is of considerable importance because it illustrates some relevant topics related to the use of MR imaging quantitative biomarkers. The paper established a clear relationship between ADC measurements and lymph node involvement. Quantitative ADC parameters derived from DW-MR images are used as imaging biomarkers because the acquisition is noninvasive, exogenous contrast agents are not require, does not use ionizing radiation, can be obtained relatively rapidly and are easily incorporated into routine patient evaluations [3]. However, some important points should be considered further.

In lymph node evaluation, the use of DW-MR images may be of relevance if an accurate relationship between malignant involvement and ADC values can be established. Authors showed that this relationship exists when using the minimum ADC, having a relatively high sensitivity $(86 \%)$, specificity $(80 \%)$ and accuracy $(82 \%)$. To be used in clinical practice, the specificity of a test (how accurately it diagnoses a particular disease without giving false-positive results) should also satisfy the clinical expectations. In this sense, $80 \%$ specificity, although high, may not be enough by itself and any effort should be made to increase the value. As DW-MR images are also used to determine depth of invasion in cervical cancer, any improvement in specificity will increase the usefulness of the technique. How to increase its diagnostic accuracy remains one of the main issues of DW-MR imaging.

Once the feasibility of DW-MR imaging in lymph node assessment has been successfully demonstrated, some comments should be made before starting to use DW-MR imaging in this setting.

To truly realize its potential, it is imperative that the DWMR technique becomes robust enough so as to provide similar information at different institutions using differing equipment. In this sense, it is still not clear which are the optimal set of b-values, which is the best curve fitting approach for the raw signal intensity data and which is the influence of the tumour type and site on the ADC values.

For cervical cancer lymph node staging, some methodological biases need to be considered and evaluated. In this paper, image acquisition was performed with a voxel size in acquisition of $3.2 \times 3.2 \times 4 \mathrm{~mm}$. Although a smaller voxel is preferred to decrease partial volume effects, acquiring with higher resolution also decreases the signal to noise ratio while facilitating the evaluation of smaller lymph nodes. Any method to increase the signal to noise ratio must be used in the evaluation of small pelvis lymph nodes.

DW-MR images were obtained with only $2 \mathrm{~b}$-values ( 0 and $1,000 \mathrm{~s} / \mathrm{m}^{2}$ ). Although this is a frequently used strategy, it can be surely improved. The trace ADC calculated values were obtained after a monoexponential fitting and this does not follow the signal behavior in tumours. This simple approach is heavily dependent on the larger b-value and does not take into consideration the (at least) biexponential behavior of the signal decay in DW-MR experiments. An intravoxel-incoherent motion (IVIM) technique with several b-values (at least 10 values) will probably enhance the accuracy of the ADC measurements. Even more, the perfusion-diffusion components $\left(\mathrm{D}, \mathrm{D}^{*}, \mathrm{f}\right)$ of the signal can be differentiated and measure together with a more accurate ADC value (Fig. 1). If the largest b-value is high enough, such as $1,700 \mathrm{~s} / \mathrm{mm}^{2}$, the IVIM technique will give more robust $\mathrm{ADC}$ values which most probably would be more reproducible among MR equipments with similar field strengths.

For the clinical implementation, the different DW-MR images obtained with the main directions and b-values should be co-registered to avoid mispositioning before the diffusion parameters are obtained. The designed fat suppression
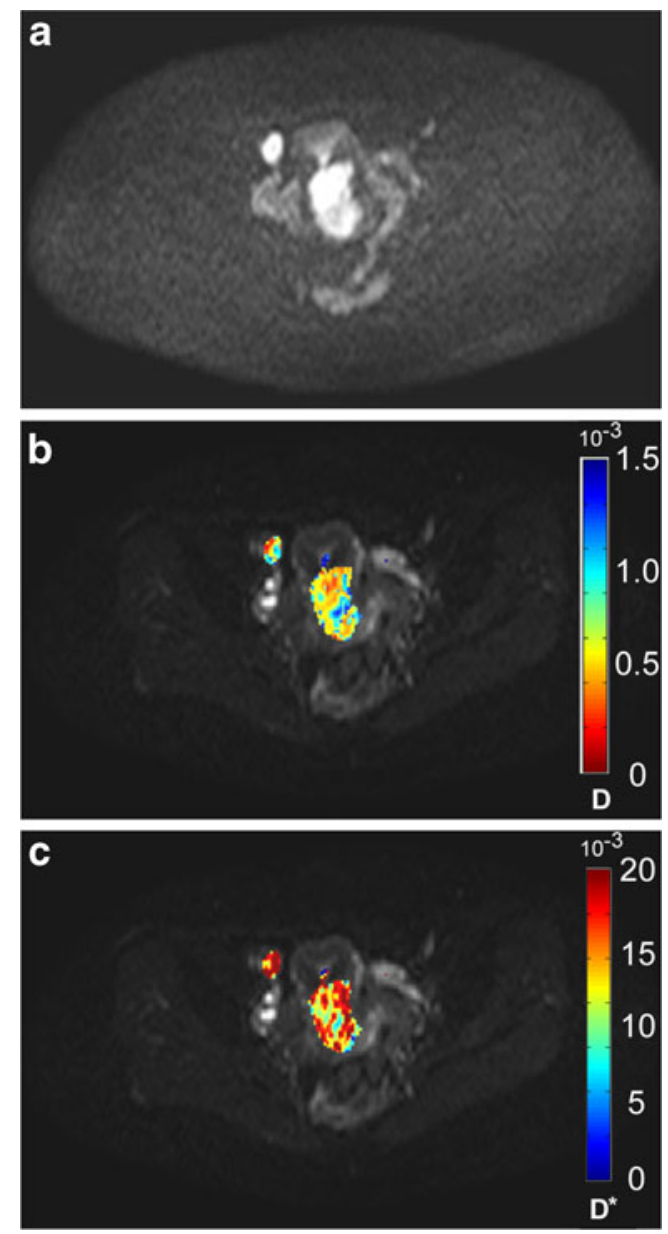

Fig. 1 Isotropic diffusion weighted image acquired using a b-value= $1,000 \mathrm{~s} / \mathrm{mm}^{2}$ (a) in a patient with cervical cancer and metastatic lymph node. Brighter areas correspond to tumor and lymphadenopathy because the high restriction of the water molecules movement. The parametric map of the diffusion component (D) superimposed on the $\mathrm{b}=0$ image (b) demonstrates pure restricted diffusion areas in red. The parametric map of the perfusion component $\left(D^{*}\right)$ superimposed on the $\mathrm{b}=0$ image (c) demonstrates the different perfusion behavior with high lesion heterogeneity. Red areas relate to increased vascular components. Note that vascularity and interstitial-intracellular water diffusion have different behavior 
strategy should be based on a spectral selective technique, such as spectral pre-saturation with inversion recovery (SPIR), in order to obtain a higher signal-to-noise ratio with reduced acquisition times. Furthermore, this approach has to be proven in large series of cases (or phantoms) evaluated at different centers. Even more, a multivariate statistical parametric analysis after combining the IVIM DW-MR imaging variables (D, $D^{*}$, and $\mathrm{f}$ ) may be of help in differentiating malignant form non-malignant lymph nodes.

Image processing has to incorporate also some type of lymph node segmentation. Authors used a manually defined ROI on the DWI or the ADC map. However, also the nodes not seen in the DW-MR images should have been measured to precisely calculate the false negative rate. A definition of how the lymph node is measured seems critical. As manually defined ROIs introduce an operator related bias, a more robust automatic threshold should be implemented in the calculated parametric maps to measure nodes by using the information from all the b-values.

The minimum ADC measurement is quite promising as the reference value. Although most authors evaluate the mean $\mathrm{ADC}$, it is quite clear that the minimum ADC criteria is better to discriminate normal from abnormal nodes, as not all of the lymph node is involved by tumour and tumour may also show necrosis with less ADC restriction. In this paper, the best $\mathrm{ADC}$ value to demonstrate involvement was the minimum, which reflects the tumour-related restriction. In order to avoid operator dependence, the complete histogram analysis of the lymph node ADC values, with the $25 \%$ percentile minimum values as the extracted parameter, should be considered when evaluating this parameter to improve accuracy.

Lymph nodes can be hyperplasic without malignant infiltration, also reducing the ADC values. The proper evaluation of ADC as a diagnostic tool should also included a larger series of cases with benign lymphadenopathy. Only then the accuracy will be properly evaluated. A larger series of cases in routine conditions with pathologic analysis is now clearly needed to know if DW-MR imaging is not only a marvelous tool for lymph node detection but also for its malignant determination.

The lack of pathological analysis is a reference standard bias. A perfect reference (the gold standard) provides an error-free classification of the presence or absence of disease. The optimal strategy is to use excision histology as the reference standard, but it is not always feasible in all patients and in most clinical situations. As some malignant lymph nodes may be PET/CT-negative, the diagnostic performance of the ADC values in this paper should be taken with some caution.

Other methodologies may compete with DW-MR images in lymph node evaluation. Sentinel lymph node is the first node where primary tumour lymphatic flow drains first, and therefore the first node where cancer cells metastasize. This lymph node is the one with the highest possibility of involvement in case of metastasis from the primary tumour. According to lymphatic mapping hypothesis, if the sentinel node is biopsy proven negative in terms of metastasis, then non-sentinel nodes are expected to be also negative. However, there may be metastasis in the non-sentinel nodes even when the sentinel node is negative. This procedure requires peritumoural tissue injection of $99 \mathrm{mTC}$ (Technetium) labeled colloids and a laparoscopic gamma probe. A recent literature review has determined that sentinel node biopsy has the highest detection rate when $99 \mathrm{mTc}$ is used in combination with blue dye (97\%), and a sensitivity of $92 \%$ [4]. Assessment of sentinel nodes provides an opportunity to stage patients intraoperatively and avoid complete pelvic lymph node dissection and hence its morbidities [5]. However, a truly preoperative assessment seems mandatory.

Lymphographic MR imaging can be obtained after ultrasmall superparamagnetic iron oxide (USPIO) administration, as this contrast agent slowly accumulates in macrophages of the normal lymph nodes. In animal models, a ROC analysis showed that USPIO enhanced MR imaging had a significantly higher diagnostic accuracy for depicting pelvic metastatic lymph nodes than did PET/ CT (area under the ROC curve, 0.984 vs $0.852 ; P=.023$ ) [6]. The results are even better if higher field strength (such as $3 \mathrm{~T}$ ) is used. Even more important although already not evaluated, the combination of SPIO-enhanced and DW-MR imaging may join the advantages of both techniques for the lymph node evaluation. Unfortunately, these agents are no longer available for clinical use.

It is important to increase and support studies that will strengthen the use of preoperative imaging to decrease the main weaknesses of these methods, among which there are detection of micrometastases, imperfect detection rate and differentiation of reactive change within a lymph node. If these objectives are met in a safe, non invasive and fast manner, accurate lymph node staging will be even more widely used in routine clinical practice. Before, advancement of a biomarker must be demonstrated in multicentre trials with standardized acquisition techniques and appropriate quality controls.

Acknowledgment Angel Alberich-Bayarri, biomedical engineer at the Radiology Department of Hospital Quirón Valencia, has greatly contributed to the manuscript reviewing.

\section{References}

1. Choi EK, Kim JK, Choi HJ, Park SH, Park BW, Kim N, Kim JS, Im KC, Cho G, Cho KS (2009) Node-by-node correlation between $\mathrm{MR}$ and PET/CT in patients with uterine cervical cancer: diffusion- 
weighted imaging versus size-based criteria on T2WI. Eur Radiol 19:2024-2032, PubMed

2. Whittaker CS, Coady A, Culver L, Rustin G, Padwick M, Padhani AR (2009) Diffusion-weighted MR imaging of female pelvic tumours: a pictorial review. Radiographics 29:759-774, PubMed

3. Padhani AR, Liu G, Koh DM, Chenevert TL, Thoeny HC, Takahara T, Dzik-Jurasz A, Ross BD, Van Cauteren M, Collins D, Hammoud DA, Rustin GJ, Taouli B, Choyke PL (2009) Diffusion-weighted magnetic resonance imaging as a cancer biomarker: consensus and recommendations. Neoplasia 11:102125, PubMed
4. van de Lande J, Torrenga B, Raijmakers PG, Hoekstra OS, van Baal MW, Brölmann HA, Verheijen RH (2007) Sentinel lymph node detection in early stage uterine cervix carcinoma: a systematic review. Gynecol Oncol 106:604-613, PubMed

5. Rasty G, Hauspy J, Bandarchi B (2009) Assessment of sentinel lymph node in cervical cancer: review of literature. J Clin Pathol 62:1062-1065, PubMed

6. Choi SH, Moon WK, Hong JH, Son KR, Cho N, Kwon BJ, Lee JJ, Chung JK, Min HS, Park SH (2007) Lymph node metastasis: ultrasmall superparamagnetic iron oxide-enhanced MR imaging versus PET/CT in a rabbit model. Radiology 242:137-143, PubMed 